\title{
VIABILIDADE ECONÔMICA E ROTAÇÃO FLORESTAL DE PLANTIOS DE CANDEIA (Eremanthus erythropappus), EM CONDIÇÕES DE RISCO
}

Carolina Souza Jarochinski e Silva ${ }^{1}$, Antonio Donizette de Oliveira ${ }^{2 *}$, Luiz Moreira Coelho Junior ${ }^{3}$, José Roberto Soares Scolforo ${ }^{2}$, Álvaro Nogueira de Souza ${ }^{4}$

*Autor para correspondência: donizete@dcf.ufla.br

RESUMO: Objetivou-se neste estudo analisar a viabilidade econômica e determinar a rotação econômica de plantios de candeia em diversos espaçamentos, em condições de risco. O estudo foi realizado a partir de um experimento de plantio de candeia constituído de quatro espaçamentos (1,5 x 1,5 m; 1,5 x 2,0 m; 1,5 x 2,5 m e 1,5 x 3,0 m), para os quais se obtiveram os fluxos de caixa relacionados às diversas idades de corte. Para a análise de risco utilizou-se o método de Monte Carlo, tendo como variável de saída (output) o Valor Anual Equivalente (VAE) e, como variáveis de entrada (fontes de incertezas), as distribuições de probabilidade referentes ao preço da muda, da terra e da madeira, o custo de colheita, a taxa de juros e a produção de madeira. A simulação constitui na realização 50.000 de iterações, de onde foram extraídas as informações necessárias às análises. Concluiu-se que o espaçamento $1,5 \times 3,0 \mathrm{~m}$ foi o mais viável economicamente e teve um nível de risco menor que os outros espaçamentos. A rotação econômica foi de $12,13,13$ e 15 anos, para o espaçamento $1,5 \times 3,0 \mathrm{~m} ; 1,5 \times 2,5 \mathrm{~m} ; 1,5 \times 2,0 \mathrm{~m}$ e 1,5 x 1,5 m, respectivamente. As informações obtidas acerca dos riscos econômicos envolvidos no plantio de candeia servem como ferramenta de auxílio na tomada de decisões em relação a novos plantios dessa espécie e também como base para futuros experimentos com a mesma, visando o aprimoramento de seu cultivo

Palavras-chave: Análise de risco. Simulação de Monte Carlo. Custo de produção.

\section{ECONOMIC VIABILITY AND ROTATION OF FORESTRY PLANTATIONS OF CANDEIA (Eremanthus erythropappus), UNDER CONDITIONS OF RISK}

\begin{abstract}
The general objective of this paper was studying the economic feasibility and determining the economic rotation of candeia planting at various spacings under risky conditions. The study was conducted from an experimental planting of candeia consisting of four spacings $(1.5 \times 1.5 \mathrm{~m}, 1.5 \times 2.0 \mathrm{~m}, 1.5 \times 2.5 \mathrm{~m}$ and $1.5 \times 3.0 \mathrm{~m})$ for which the cash flows related to the different cutting ages were obtained. For the risk analysis the Monte Carlo method was used, its having the Equivalent Annual Value (EAV) as the output variable (output) and as input variables (sources of uncertainty) the probability distributions concerning the price of the seedlings, land and wood, the harvest cost, interest rates and timber production. The simulation constituted in the doing of 50,000 iterations from where the information necessary to the analyses was extracted. It was concluded that the $1.5 \times 3.0 \mathrm{~m}$ spacing was the most economically viable and presented a lower risk level than the other spacings. The economic rotation was 12, 13, 13 and 15 years for the spacings $1.5 \times 3.0 \mathrm{~m}, 1.5 \times 2.5 \mathrm{~m}, 1.5 \times 2.0 \mathrm{~m}$ and $1.5 \times 1.5 \mathrm{~m}$, respectively. Information obtained about the economic risks involved in planting candle serve as a tool to aid in making decisions regarding new plantings of this species and also as a basis for future experiments with the same, seeking to improve its culture.
\end{abstract}

Key words: Risk analysis. Monte Carlo simulation. Production cost

\section{INTRODUÇÃO}

A candeia (Eremanthus erythropappus) é uma espécie nativa do Brasil e com grande ocorrência no estado de Minas Gerais. Ela fornece multiprodutos, como moirão de cerca com alta durabilidade natural e matéria prima de onde se extrai um óleo essencial, cujo principal componente (alfabisabolol) possui importantes propriedades medicinais e grande aplicação na indústria de

${ }^{1}$ Fibria - Aracruz, Espírito Santo, Brasil.

${ }^{2}$ Universidade Federal de Lavras - Lavras, Minas Gerais, Brasil

${ }^{3}$ Instituto Agronômico do Paraná - Santa Tereza do Oeste, Paraná, Brasil

${ }^{4}$ Universidade de Brasília - Brasília, Distrito Federal, Brasil cosméticos e medicamentos. Esses multiprodutos servem como fonte alternativa de renda para os proprietários rurais de suas regiões de ocorrência.

Características particulares da espécie, como ocorrência em solos pouco férteis e em áreas de elevada altitude (800 a $1.500 \mathrm{~m}$ ), lhe conferem a vantagem de se desenvolver em locais onde seria difícil a implantação de culturas agrícolas ou de outras espécies florestais.

Aproveitando suas peculiaridades e visando

Cerne, Lavras, v. 20, n. 1, p. 113-122, jan./mar. 2014 
minimizar a exploração de candeais nativos, os plantios de candeia surgem como uma interessante opção de cultivo florestal. Mas, para garantir a segurança financeira de um empreendimento inovador como esse é necessário conhecer sua viabilidade econômica. Isso pode ser executado por meio de uma análise acerca dos custos e receitas envolvidos no plantio, conhecida como fluxo de caixa. Ocorre que esse fluxo é repleto de incertezas, devido à inexistência de valores plenamente confiáveis. Segundo Lapponi (2007), entre as causas mais prováveis dos desvios desfavoráveis do projeto está o erro de estimativa provocado pela falta de experiência, pela incorreta pesquisa de mercado, pelos custos e receitas menores ou maiores que os estimados, pela escolha inadequada de tecnologia, pela habilidade gerencial, pelo ambiente econômico, etc.

Segundo Securato (2007), um evento que com certeza ocorrerá tem uma probabilidade de $100 \%$ e, diante de eventos que apresentem certo grau de incerteza, é possível estabelecer a probabilidade de ocorrência deste evento. Para Mendes e Souza (2007), esse grau de incerteza a respeito de um evento pode também ser chamado de risco. E a análise quantitativa do risco, usando a simulação de Monte Carlo, oferece ao usuário um método poderoso e preciso para abordar as várias incertezas associadas às atividades de um empreendimento.

A aplicação de análise de risco vem sendo cada vez mais incorporada ao setor florestal (BENTES-GAMA et al., 2005; CASTRO et al., 2007; COELHO JÚNIOR; et al., 2008; CORDEIRO; SILVA, 2010; KALLIO, 2010; KNOKE; MOOG; PLUSCZYK, 2001). Isso se deve ao fato de que a produção florestal tem características de investimentos de médio a longo prazo que envolvem um alto capital imobilizado na implantação do projeto. Além disso, os produtos florestais como fenômenos biológicos não são eventos determinísticos, mas sim probabilísticos, pois sua produtividade envolve sempre um grau de risco ou incerteza (COELHO JÚNIOR et al., 2008).

Objetivou-se, neste estudo, analisar a viabilidade econômica e determinar a rotação econômica em plantios de candeia (Eremanthus erythropappus) em diversos espaçamentos, em condições de risco.

\section{MATERIAL E MÉTODOS}

\section{1 Área de estudo}

A área de estudo situa-se no município de Carrancas que está localizado no sul de Minas Gerais, nas coordenadas $-21,48^{\circ} \mathrm{S}$ e $-44,64^{\circ} \mathrm{O}$ (INSTITUTO BRASILEIRO DE GEOGRAFIA E ESTATÍSTICA IBGE, 2011). O município apresenta uma área de 728,5 $\mathrm{km}^{2}$ de extensão e altitude variando de $896 \mathrm{~m}$ a $1.590 \mathrm{~m}$ (ASSEMBLÉIA LEGISLATIVA DE MINAS GERAIS ALMG, 2011).

O clima do município de Carrancas, segundo a classificação de Köppen, é do tipo Cwa, com temperaturas moderadas e verão quente e chuvoso. As médias anuais de precipitação e a temperatura são de $1.470 \mathrm{~mm}$ e $19,2^{\circ} \mathrm{C}$, respectivamente (ALMG, 2011).

$\mathrm{O}$ experimento em estudo trata-se de um plantio de candeia instalado em março de 2002, com área de 2,87 ha em quatro blocos casualizados divididos em quatro parcelas casualizadas e, em cada uma delas, plantaramse 532 covas em 14 linhas, nos seguintes espaçamentos: tratamento 1 (T1) - espaçamento $1,5 \times 1,5 \mathrm{~m}$; tratamento 2 (T2)- espaçamento $1,5 \times 2,0 \mathrm{~m}$; tratamento 3 (T3)espaçamento $1,5 \times 2,5 \mathrm{~m}$; tratamento 4 (T4)- espaçamento $1,5 \times 3,0 \mathrm{~m}$.

A adubação de plantio foi feita com $100 \mathrm{~g} /$ cova do adubo formulado NPK na composição 4-14-8 + Zn-0,4\%. Já na adubação de cobertura utilizou-se 30g/cova de Bórax. Para o combate às formigas, utilizou-se isca granulada.

\subsection{Estimativa do volume de madeira para as diversas idades do plantio de candeia}

Os volumes de madeira para as diferentes idades do plantio de candeia foram obtidos do estudo de Silva (2009) que ajustou modelos de crescimento para os diversos tratamentos desse experimento. Nesse estudo, o volume de madeira foi estimado em metro cúbico. Para se obter os valores da Tabela 1 que estão expressos em metro estéreo (mst) utilizou-se um fator de empilhamento igual a 2,67, o qual foi determinado por Pérez (2001).

Tabela 1 - Volume de madeira (mst/ha) para as diversas idades e tratamentos.

Table 1 - Wood volume (mst/ha) for different ages and treatments.

\begin{tabular}{ccccc}
\hline & $\mathrm{T} 1$ & $\mathrm{~T} 2$ & $\mathrm{~T} 3$ & $\mathrm{~T} 4$ \\
Idade & $(1,5 \times 1,5 \mathrm{~m})$ & $(1,5 \times 2,0 \mathrm{~m})$ & $(1,5 \times 2,5 \mathrm{~m})$ & $(1,5 \times 3,0 \mathrm{~m})$ \\
\hline 10 & 74,73 & 77,86 & 67,66 & 71,13 \\
11 & 82,18 & 85,63 & 74,39 & 78,20 \\
12 & 89,63 & 93,37 & 81,11 & 85,31 \\
13 & 97,08 & 101,11 & 87,84 & 92,38 \\
14 & 104,50 & 108,86 & 94,57 & 99,46 \\
15 & 111,93 & 116,60 & 101,30 & 106,53 \\
\hline
\end{tabular}

Cerne, Lavras, v. 20, n. 1, p. 113-122, jan./mar. 2014 


\subsection{Custos e receitas relacionados ao plantio da candeia}

Os custos relacionados às atividades de implantação (preparo do solo, plantio, mudas, adubação, combate a formigas, etc.) e de manutenção (combate a formigas, capina, etc.) do plantio da candeia estão sendo registrados desde 2002, quando o experimento foi implantado. Entretanto, estes valores serviram apenas como referência para elaborar a planilha de custos a ser utilizada na análise econômica, uma vez que, por se tratar de um experimento, alguns deles podem estar superestimados em função do maior tempo requerido para a execução de determinadas atividades inerentes à implantação e à manutenção do experimento.

A planilha de custos utilizada na análise econômica (Tabela 2) foi elaborada da seguinte maneira:

- custos das atividades e dos insumos necessários à implantação de candeia (até 6 meses de idade): foram obtidos por meio de levantamentos de dados em propriedades rurais situadas nos municípios de Baependi, Caxambú e Carrancas, etc. que participam do programa de fomento para o plantio de candeia financiado pelo Instituto Estadual de Florestas de Minas Gerais (IEF-MG).

- custos de manutenção anual até a idade de corte: foram estimados usando como referência os custos de manutenção de plantios de eucalipto, uma vez que os plantios de candeia existentes ainda são jovens e, portanto, não se dispõe de informações relacionadas às manutenções anuais dessa espécie. Assume-se, nesse caso, que a manutenção do candeal plantado é semelhante à de um povoamento de eucalipto manejado para a produção de madeira para energia ou celulose;

- custo de colheita, que envolve a derrubada, o traçamento e a extração da madeira até a beira da estrada: foi considerado como sendo igual a $\mathrm{R} \$ 25,18 /$ mst, baseando-se no estudo de Oliveira et al. (2010), que o determinaram para a exploração de candeia nativa. Para se obter o custo de colheita por hectare para cada tratamento nas diversas idades, multiplicou-se esse valor pelos volumes de madeira apresentados na Tabela 1 .

- custo anual da terra: foi considerado como sendo os juros sobre o valor desse fator de produção. $\mathrm{O}$ valor da terra considerado foi de $\mathrm{R} \$ 2.500,00 /$ ha, que é o valor praticado na região.

As receitas foram obtidas multiplicando-se o volume de madeira estimado para cada espaçamento nas diversas idades (Tabela 1) pelo seu preço de venda colocada na beira da estrada, que foi considerado como sendo de R \$ 130,00/mst. Esse valor foi obtido por meio de entrevistas realizadas com agricultores de municípios que vendem madeira de candeia nativa para as fábricas de óleo, e entrevistas com os gerentes das empresas produtoras de óleo de candeia que compram madeira oriunda de candeais situados em Minas Gerais.

Tabela 2- Custos das atividades de implantação e manutenção de candeia, para os quatro tratamentos estudados.

Table 2 - Plantation and maintenance activity cost of candeia for the four treatments studied.

\begin{tabular}{|c|c|c|c|c|c|}
\hline \multirow[t]{2}{*}{ Itens de Custo } & \multirow{2}{*}{$\begin{array}{c}\text { Ano de } \\
\text { ocorrência }\end{array}$} & \multicolumn{4}{|c|}{ Valor do Custo (R $\$ / \mathrm{ha})$} \\
\hline & & $\mathrm{T} 1$ & $\mathrm{~T} 2$ & T3 & $\mathrm{T} 4$ \\
\hline \multicolumn{6}{|l|}{ 1. Implantação } \\
\hline $\begin{array}{l}\text { Preparo do solo (sul- } \\
\text { cagem) }\end{array}$ & 0 & 200,00 & 200,00 & 200,00 & 200,00 \\
\hline $\begin{array}{l}\text { Mão de obra de } \\
\text { plantio }\end{array}$ & 0 & 559,92 & 420,00 & 336,00 & 279,96 \\
\hline Adubação de plantio & 0 & 319,57 & 239,72 & 191,77 & 159,79 \\
\hline $\begin{array}{l}\text { Adubação de co- } \\
\text { bertura }\end{array}$ & 0 & 260,36 & 30 & 15 & 130,18 \\
\hline Mudas & 0 & $2.333,00$ & $1.750,00$ & $1.400,00$ & $1.166,50$ \\
\hline $\begin{array}{l}\text { Combate às for- } \\
\text { migas }\end{array}$ & 0 & 17,00 & 17,00 & 17,00 & 17,00 \\
\hline \multicolumn{6}{|l|}{ 2. Manutenção } \\
\hline $\begin{array}{l}\text { Capina e roçada } \\
\text { (manual) }\end{array}$ & 1 & 210,00 & 210,00 & 210,00 & 210,00 \\
\hline $\begin{array}{l}\text { Capina e roçada } \\
\text { (manual) }\end{array}$ & 2 a 4 & 90,00 & 90,00 & 90,00 & 90,00 \\
\hline $\begin{array}{l}\text { Combate às for- } \\
\text { migas }\end{array}$ & 1 a 4 & 13,50 & 13,50 & 13,50 & 13,50 \\
\hline Custo da terra & $1 \mathrm{an}$ & 150,00 & 150,00 & 150,00 & 150,00 \\
\hline
\end{tabular}

\subsection{Variável de saída (output) do modelo}

A variável de saída do modelo (output) é o resultado da simulação e, a partir dela, é feita a tomada de decisões em relação à viabilidade econômica do plantio da candeia em diversos espaçamentos e à determinação de sua rotação econômica, em condições de risco. Neste estudo utilizou-se como variável de saída o Valor Anual Equivalente (VAE) ou Benefício (Custo) Periódico Equivalente (B(C)PE) que, segundo Rezende e Oliveira (2008), é dado pela equação 1:

$$
\begin{aligned}
& V A E=\frac{V P L . i .(1+i)^{n}}{(1+i)^{n}-1} \\
& \text { Onde: } \\
& V P L=\sum_{j=0}^{n} R_{j}(1+i)^{-j}-C_{j}(1+i)^{-j} \sum_{j=0}^{n}
\end{aligned}
$$

Cerne, Lavras, v. 20, n. 1, p. 113-122, jan./mar. 2014 
$C_{j}=$ custo ao final do ano $j$;

$R_{j}=$ receita ao final do ano $j$;

$i=$ taxa de juros ou de desconto;

$n=$ idade do plantio, em anos.

Determinou-se o VAE para os quatro tratamentos nas idades de 10 a 15 anos. A rotação econômica foi definida como sendo a idade em que se obteve o maior VAE e o menor risco.

\subsection{Variáveis de entrada (input) do modelo}

As variáveis de entrada (inputs) ou variáveis de risco são valores desconhecidos ou incertos que devem ser modelados como distribuições de probabilidade. As estimativas dos custos e receitas relacionadas ao cultivo da candeia são consideradas uma fonte de riscos, pois existem incertezas em relação a esses valores. Assim, neste estudo, consideraram-se as seguintes variáveis de risco: preço da muda ( $R$ \$), custo anual da terra ( $R$ \$/ha), custo de colheita (R\$/mst), taxa de juros (a.a.), preço de venda da madeira ( $\mathrm{R} \$ \mathrm{mst}$ ) e produção volumétrica de madeira ( $\mathrm{mst} / \mathrm{ha})$.

A distribuição de probabilidade utilizada para representar essas variáveis foi a triangular e os valores mínimos, mais prováveis e máximos utilizados encontramse nas Tabelas 3 e 4.

Os valores máximos e mínimos referentes à produção volumétrica foram determinados considerandose uma produção volumétrica de $10 \%$ para mais e para

Tabela 3 - Valores utilizados para a determinação da distribuição triangular das variáveis de entrada utilizadas na simulação de Monte Carlo.

Table 3 - Values used for the determination of the triangular distribution of the input variables used in the Monte Carlo simulation.

\begin{tabular}{lccccc}
\hline \multicolumn{1}{c}{$\begin{array}{c}\text { Itens de } \\
\text { custos }\end{array}$} & Unidade & $\begin{array}{c}\text { Variável } \\
\text { de } \\
\text { entrada }\end{array}$ & $\begin{array}{c}\text { Valor } \\
\text { mínimo }\end{array}$ & $\begin{array}{c}\text { Valor } \\
\text { mais } \\
\text { provável }\end{array}$ & $\begin{array}{c}\text { Valor } \\
\text { máximo }\end{array}$ \\
\hline $\begin{array}{l}\text { Preço das } \\
\text { mudas }\end{array}$ & $\begin{array}{c}\mathrm{R} \$ 1 \\
\text { muda }\end{array}$ & 0,50 & 0,40 & 0,50 & 0,60 \\
$\begin{array}{l}\text { Valor da } \\
\text { terra }\end{array}$ & $\mathrm{R} \$ / \mathrm{ha}$ & $2.500,00$ & $2.000,00$ & $2.500,00$ & $3.000,00$ \\
$\begin{array}{l}\text { Custo de } \\
\text { colheita }\end{array}$ & $\mathrm{R} \$ / \mathrm{mst}$ & 25,18 & 20,18 & 25,18 & 30,18 \\
$\begin{array}{l}\text { Taxa de juros } \\
\begin{array}{l}\text { Preço de } \\
\text { venda da } \\
\text { madeira }\end{array}\end{array}$ & a.a. $\$ / \mathrm{mst}$ & 130,00 & 115,00 & 130,00 & 145,00 \\
\hline
\end{tabular}

Cerne, Lavras, v. 20, n. 1, p. 113-122, jan./mar. 2014 menos em relação aos volumes obtidos por Silva (2009), mostrados na Tabela 1.

Segundo Souza (2001), as distribuições triangular e uniforme, geralmente, são muito utilizadas nas ciências agrárias e na economia, por apresentarem simplicidade e a vantagem de não necessitarem de muitos dados de um determinado evento.

Tabela 4 - Valores de produção volumétrica utilizados na simulação de Monte Carlo.

Table 4 - Values of volumetric production used in the Monte Carlo simulation.

\begin{tabular}{|c|c|c|c|c|}
\hline \multirow[b]{2}{*}{ Idades } & \multicolumn{4}{|c|}{ Tratamento $1(1,5 \times 1,5 \mathrm{~m})$} \\
\hline & $\begin{array}{c}\text { Variável de } \\
\text { entrada }\end{array}$ & Valor mínimo & $\begin{array}{c}\text { Valor mais } \\
\text { provável }\end{array}$ & Valor máximo \\
\hline 10 & 74,73 & 67,26 & 74,73 & 82,21 \\
\hline 11 & 82,18 & 73,96 & 82,18 & 90,40 \\
\hline 12 & 89,63 & 80,67 & 89,63 & 98,60 \\
\hline 13 & 97,08 & 87,37 & 97,08 & 106,79 \\
\hline 14 & 104,50 & 94,05 & 104,50 & 114,95 \\
\hline 15 & 111,93 & 100,73 & 111,93 & 123,12 \\
\hline \multirow[b]{2}{*}{ Idades } & \multicolumn{4}{|c|}{ Tratamento $2(1,5 \times 2,0 \mathrm{~m})$} \\
\hline & $\begin{array}{c}\text { Variável de } \\
\text { entrada }\end{array}$ & Valor mínimo & $\begin{array}{c}\text { Valor mais } \\
\text { provável }\end{array}$ & Valor máximo \\
\hline 10 & 77,86 & 70,10 & 77,86 & 85,64 \\
\hline 11 & 85,63 & 77,10 & 85,63 & 94,19 \\
\hline 12 & 93,37 & 84,03 & 93,37 & 102,71 \\
\hline 13 & 101,11 & 91,00 & 101,11 & 111,22 \\
\hline 14 & 108,86 & 97,97 & 108,85 & 119,74 \\
\hline 15 & 116,60 & 104,94 & 116,60 & 128,26 \\
\hline \multirow[b]{2}{*}{ Idades } & \multicolumn{4}{|c|}{ Tratamento $3(1,5 \times 2,5 \mathrm{~m})$} \\
\hline & $\begin{array}{c}\text { Variável de } \\
\text { entrada }\end{array}$ & Valor mínimo & $\begin{array}{c}\text { Valor mais } \\
\text { provável }\end{array}$ & Valor máximo \\
\hline 10 & 67,66 & 60,89 & 67,66 & 74,42 \\
\hline 11 & 74,39 & 66,95 & 74,39 & 81,82 \\
\hline 12 & 81,11 & 73,00 & 81,11 & 89,23 \\
\hline 13 & 87,84 & 79,06 & 87,84 & 96,63 \\
\hline 14 & 94,57 & 85,11 & 94,57 & 104,03 \\
\hline 15 & 101,30 & 91,17 & 101,30 & 111,43 \\
\hline \multirow[b]{2}{*}{ Idades } & \multicolumn{4}{|c|}{ Tratamento $4(1,5 \times 3,0 \mathrm{~m})$} \\
\hline & $\begin{array}{c}\text { Variável de } \\
\text { entrada }\end{array}$ & Valor mínimo & $\begin{array}{c}\text { Valor mais } \\
\text { provável }\end{array}$ & Valor máximo \\
\hline 10 & 71,13 & 64,02 & 71,13 & 78,24 \\
\hline 11 & 78,20 & 70,38 & 78,20 & 86,02 \\
\hline 12 & 85,31 & 76,78 & 85,31 & 93,84 \\
\hline 13 & 92,38 & 83,14 & 92,38 & 101,62 \\
\hline 14 & 99,46 & 89,51 & 99,46 & 109,40 \\
\hline 15 & 106,53 & 95,88 & 106,53 & 117,19 \\
\hline
\end{tabular}




\subsection{Simulação e análise dos dados}

A análise de risco foi realizada pela aplicação do método de Monte Carlo, com o auxílio do software @ RISK (PALISADE CORPORATION, 2009). A simulação de Monte Carlo é um processo que possibilita imitar uma realidade por meio de modelos e as simulações por processos aleatórios possibilitam lidar com situações cuja evolução, no decorrer do tempo, não seja previsível, trabalhando com eventos aleatórios ou probabilísticos (quando sua ocorrência envolve certo risco ou grau de incerteza).

Primeiramente, foi realizada uma simulação automática pelo software, visando monitorar a convergência. Em seguida, realizou-se uma segunda simulação com o número ideal de iterações, que é acima daquele em que houve convergência.

O número de iterações utilizado foi de 50.000. A partir das iterações realizadas, foram gerados os resultados relacionados à variável de saída do modelo, como distribuições de probabilidade, frequências acumulada e relativa, estatísticas descritivas, etc.

\section{RESULTADOS E DISCUSSÃO}

\subsection{Análise de risco}

Para cada tratamento e em cada idade geraram-se, por meio da simulação de Monte Carlo, 50.000 valores para o indicador econômico Valor Anual Equivalente (VAE) (Tabela 5). No tratamento 1, o VAE esperado, ou VAE médio, foi negativo em todas as idades, e o menor valor negativo ocorreu aos 15 anos (R $\$-79,25)$, sendo, portanto, essa a idade ótima de corte ou rotação econômica. A informação de que o VAE esperado é negativo a princípio indica que o tratamento é inviável. Porém, a avaliação sob condições de incerteza é relativa. Assim, ao analisar os percentis gerados para este tratamento, observa-se que, a partir dos 11 anos, existem probabilidades, mesmo que pequenas, de se obter VAEs positivos. Para a idade de 15 anos, há $15,1 \%$ de chances de que o VAE seja positivo, ou seja, trata-se de um investimento de alto risco, mas com chances de ser viável economicamente.

O maior VAE dos tratamentos 2 e 3 ocorre aos 13 anos. Pela análise dos percentis observa-se que, nessa idade, $5 \%$ dos VAEs do tratamento 2 são maiores que $\mathrm{R} \$ 155,23$ e 5\% são menores ou iguais a - R \$ 82,96. Já no tratamento $3,5 \%$ desses valores são maiores que $\mathrm{R} \$ 126,25$ e $5 \%$ são menores ou iguais $R \$-84,44$. As chances de ocorrerem VAEs negativos, cortando-se a candeia aos 13 anos de idade, são de $32,3 \%$ para o tratamento 2 e de $38,9 \%$ para o tratamento 3 .

No tratamento 4, o maior VAE ocorreu aos 12 anos. Se o corte da madeira for feito nessa idade, há apenas $9,1 \%$ de chances de se obter VAE negativo. Segundo Hacura, Jamadus-Hacura e Kotot (2001), geralmente, quando a probabilidade de se obter Valor Presente Líquido (VPL) negativo é menor que $20 \%$, o projeto é bastante seguro. O valor crítico dessa medida de risco é subjetivo, pois cada gerente tem um grau diferente de aversão ao risco.

Na Figura 1, observa-se a distribuição de probabilidades dos VAEs, a curva de frequência

Tabela 5 - Estatísticas descritivas do VAE para os diversos tratamentos e idades de corte.

Table 5 - Descriptive statistics of the Equivalent Annual Value (EAV) for the various treatments and cutting ages.

\begin{tabular}{|c|c|c|c|c|c|c|c|}
\hline \multicolumn{8}{|c|}{ Tratamento 1} \\
\hline Idade & $\begin{array}{c}\text { VAE } \\
\text { esperado } \\
\left(\mathrm{R} \$ \mathrm{ha}^{-1}\right)\end{array}$ & $\begin{array}{c}\text { Mínimo } \\
\left(\mathrm{R} \$ \mathrm{ha}^{-1}\right)\end{array}$ & $\begin{array}{l}\text { Máximo } \\
\left(\mathrm{R} \$ \cdot \mathrm{ha}^{-1}\right)\end{array}$ & $\mathrm{VAE}<0$ & $\begin{array}{c}5 \% \\
\left(\mathrm{R} \$ \cdot \mathrm{ha}^{-1}\right)\end{array}$ & $\begin{array}{c}50 \% \\
\left(\mathrm{R} \$ \cdot \mathrm{ha}^{-1}\right)\end{array}$ & $\begin{array}{c}95 \% \\
\left(\mathrm{R} \$ \mathrm{ha}^{-1}\right)\end{array}$ \\
\hline 10 & $-126,32$ & $-390,78$ & 179,35 & $100,00 \%$ & $-247,67$ & $-127,14$ & $-2,57$ \\
\hline 11 & $-107,42$ & $-387,98$ & 202,84 & $92,20 \%$ & $-229,33$ & $-107,89$ & 16,6 \\
\hline 12 & $-94,41$ & $-379,22$ & 201,55 & $89,40 \%$ & $-216,39$ & $-94,99$ & 29,47 \\
\hline 13 & $-86,3$ & $-349,55$ & 212 & $87,30 \%$ & $-207,71$ & $-87,14$ & 38,49 \\
\hline 14 & $-81,41$ & $-360,73$ & 229,01 & $85,80 \%$ & $-204,22$ & $-82,31$ & 43,78 \\
\hline 15 & $-79,25$ & $-364,08$ & 214,8 & $84,90 \%$ & $-201,88$ & $-80,29$ & 47,15 \\
\hline \multicolumn{8}{|c|}{ Tratamento 2} \\
\hline Idade & $\begin{array}{c}\text { VAE } \\
\text { esperado } \\
\left(\mathrm{R} \$ \cdot \mathrm{ha}^{-1}\right)\end{array}$ & $\begin{array}{l}\text { Mínimo } \\
\left(\mathrm{R} \$ \cdot \mathrm{ha}^{-1}\right)\end{array}$ & $\begin{array}{l}\text { Máximo } \\
\left(\mathrm{R} \$ \cdot \mathrm{ha}^{-1}\right)\end{array}$ & $\mathrm{VAE}<0$ & $\begin{array}{c}5 \% \\
\left(\mathrm{R} \$ . h \mathrm{~h}^{-1}\right)\end{array}$ & $\begin{array}{c}50 \% \\
\left(\mathrm{R} \$ \cdot h a^{-1}\right)\end{array}$ & $\begin{array}{c}95 \% \\
\left(\mathrm{R} \$ . \mathrm{ha}^{-1}\right)\end{array}$ \\
\hline 10 & 16,7 & $-247,87$ & 275,25 & $41,30 \%$ & $-100,28$ & 15,61 & 136,65 \\
\hline 11 & 26,6 & $-226,4$ & 305,4 & $36,10 \%$ & $-89,32$ & 25,89 & 145,51 \\
\hline 12 & 32,29 & $-219,59$ & 333,39 & $33,00 \%$ & $-84,65$ & 31,58 & 152 \\
\hline 13 & 34,1 & $-224,75$ & 325,69 & $32,30 \%$ & $-82,96$ & 32,98 & 155,23 \\
\hline 14 & 33,73 & $-217,47$ & 314,82 & $32,50 \%$ & $-83,66$ & 32,36 & 155,3 \\
\hline 15 & 31,21 & $-234,4$ & 294,21 & $34,00 \%$ & $-86,99$ & 30,28 & 152,99 \\
\hline \multicolumn{8}{|c|}{ Tratamento 3} \\
\hline Idade & $\begin{array}{c}\text { VAE } \\
\text { esperado } \\
\left(\mathrm{R} \$ \cdot \mathrm{ha}^{-1}\right)\end{array}$ & $\begin{array}{l}\text { Mínimo } \\
\left(\mathrm{R} \$ \cdot \mathrm{ha}^{-1}\right)\end{array}$ & $\begin{array}{l}\text { Máximo } \\
\left(\mathrm{R} \$ \cdot \mathrm{ha}^{-1}\right)\end{array}$ & $\mathrm{VAE}<0$ & $\begin{array}{c}5 \% \\
\left(\mathrm{R} \$ . \mathrm{ha}^{-1}\right)\end{array}$ & $\begin{array}{c}50 \% \\
\left(\mathrm{R} \$ \cdot \mathrm{ha}^{-1}\right)\end{array}$ & $\begin{array}{c}95 \% \\
\left(\mathrm{R} \$ \mathrm{ha}^{-1}\right)\end{array}$ \\
\hline 10 & 6,14 & $-242,63$ & 245,84 & $46,60 \%$ & $-98,17$ & 5,7 & 111,31 \\
\hline 11 & 13,9 & $-224,1$ & 265,21 & $41,90 \%$ & $-89,21$ & 13,12 & 119,72 \\
\hline 12 & 18,01 & $-212,59$ & 271,55 & $39,30 \%$ & $-86,87$ & 17,46 & 124,23 \\
\hline 13 & 19,33 & $-202,14$ & 266,78 & $38,90 \%$ & $-84,44$ & 18,57 & 126,25 \\
\hline 14 & 18,28 & $-218,12$ & 299,17 & $39,50 \%$ & $-86,27$ & 17,25 & 126,3 \\
\hline 15 & 15,84 & $-211,14$ & 274,16 & $41,10 \%$ & $-89,31$ & 14,71 & 123,88 \\
\hline \multicolumn{8}{|c|}{ Tratamento 4} \\
\hline Idade & $\begin{array}{c}\text { VAE } \\
\text { esperado } \\
\left(\mathrm{R} \$ \mathrm{ha}^{-1}\right)\end{array}$ & $\begin{array}{c}\text { Mínimo } \\
\left(\mathrm{R} \$ \cdot \mathrm{ha}^{-1}\right)\end{array}$ & $\begin{array}{l}\text { Máximo } \\
\left(\mathrm{R} \$ \cdot \mathrm{ha}^{-1}\right)\end{array}$ & $\mathrm{VAE}<0$ & $\begin{array}{c}5 \% \\
\left(\mathrm{R} \$ \cdot \mathrm{ha}^{-1}\right)\end{array}$ & $\begin{array}{c}50 \% \\
\left(\mathrm{R} \$ \cdot \mathrm{ha}^{-1}\right)\end{array}$ & $\begin{array}{c}95 \% \\
\left(\mathrm{R} \$ \mathrm{ha}^{-1}\right)\end{array}$ \\
\hline 10 & 80,95 & $-162,44$ & 335,64 & $10,40 \%$ & $-23,28$ & 80,18 & 187,52 \\
\hline 11 & 84,75 & $-141,05$ & 349,25 & $9,40 \%$ & $-19,93$ & 83,96 & 191,29 \\
\hline 12 & 85,74 & $-136,4$ & 321,75 & $9,10 \%$ & $-19,25$ & 85,03 & 193,83 \\
\hline 13 & 83,86 & $-146,35$ & 334,71 & $9,70 \%$ & $-21,1$ & 82,97 & 192,44 \\
\hline 14 & 80,13 & $-159,57$ & 333,64 & $10,80 \%$ & $-25,09$ & 79,22 & 188,15 \\
\hline 15 & 75,07 & $-153,87$ & 342,2 & $12,50 \%$ & $-30,47$ & 74,27 & 184,1 \\
\hline
\end{tabular}

Cerne, Lavras, v. 20, n. 1, p. 113-122, jan./mar. 2014 
acumulada (eixo da direita), a probabilidade de ocorrerem VAEs positivos e negativos nas idades correspondentes à rotação econômica de cada tratamento e a posição em que se situam os valores de VAE média, moda e mediana. $\mathrm{O}$ círculo na curva de frequência acumulada em cada figura indica a interseção entre o VAE igual a zero e sua probabilidade de ocorrência na curva de frequência acumulada. A informação fornecida por esta interseção é a de que, no tratamento 1 , por exemplo, existem $15,1 \%$ de chances de o VAE ser maior que
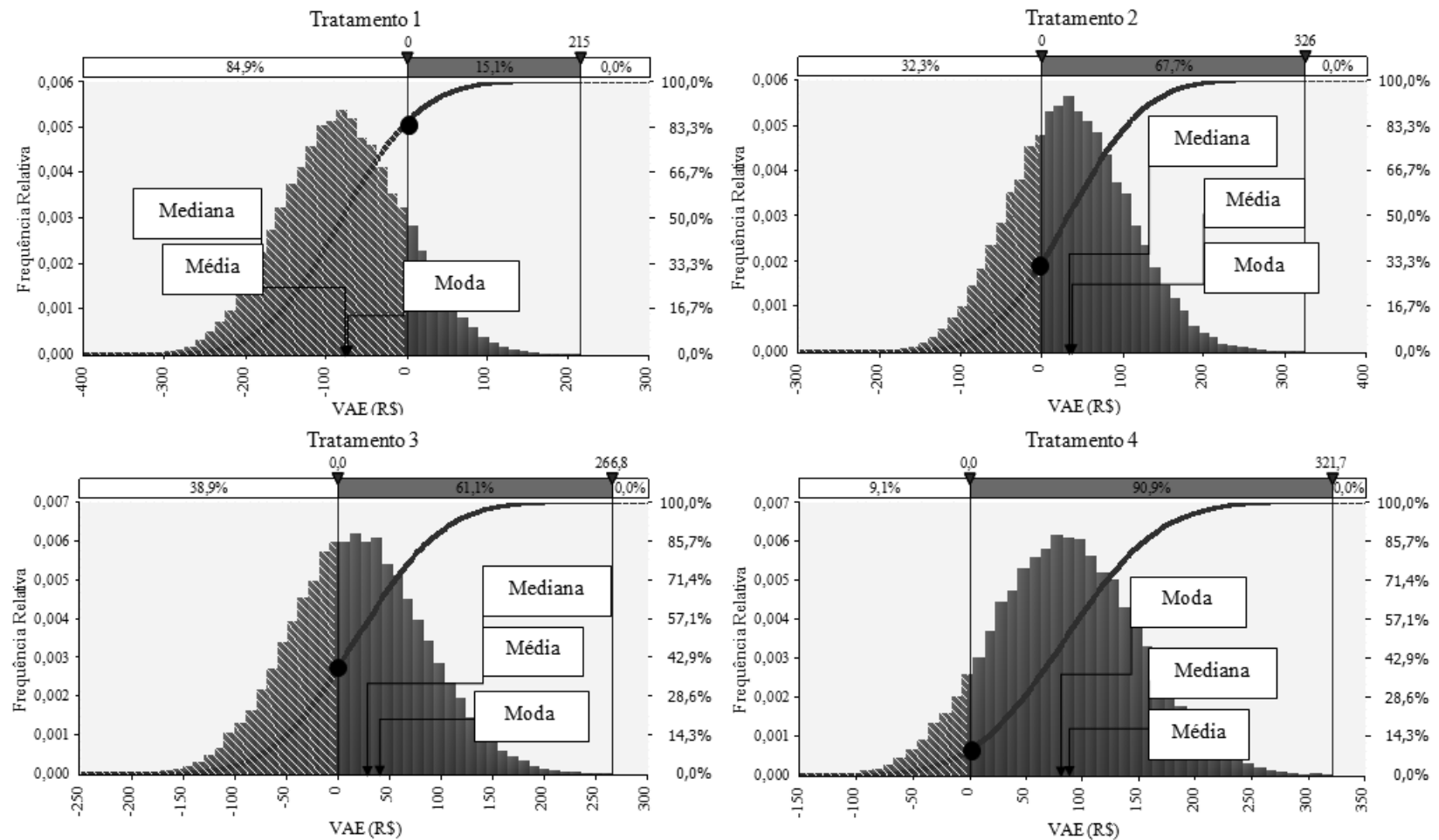

Figura 1 - Distribuição de frequência relativa e acumulada, probabilidade de ocorrência de VAEs positivos e negativos na rotação econômica de cada tratamento, posição da média, mediana e moda dos VAEs.

Figure 1 - Distribution of relative frequency and cumulative, EAV likelihood of positive and negative economic rotation of each treatment, position of the mean, median and mode of EAV.

zero (frequência acumulada ao lado direito do círculo) e, consequentemente, $84,9 \%$ de chances de o VAE ser menor que zero (frequência acumulada ao lado esquerdo do círculo).

Nota-se, na Figura 1, que os VAEs estão distribuídos simetricamente ao redor da média, com valores médios iguais ou muito próximos à mediana, ou seja, não há inclinação acentuada da distribuição dos valores para nenhum dos lados em nenhum tratamento.

Segundo Lapponi (2007), considerando que o valor esperado é equidistante dos valores da série e a mediana é um valor na posição central da mesma série ordenada de forma crescente, a comparação do valor esperado e da mediana antecipa a forma da distribuição de frequências do VPL. Quanto mais o valor esperado se distanciar da mediana, mais acentuada será a inclinação da distribuição de frequências do VPL de forma que, se o valor esperado for maior que a mediana, a distribuição terá inclinação à direita e vice-versa.

Na Figura 2 estão indicados os VAEs máximos e mínimos, o VAE esperado ou médio e os valores alcançados com um desvio padrão para mais e para menos em relação à média para a idade de rotação econômica de cada tratamento. No tratamento 4 os VAEs variam na faixa

Cerne, Lavras, v. 20, n. 1, p. 113-122, jan./mar. 2014 

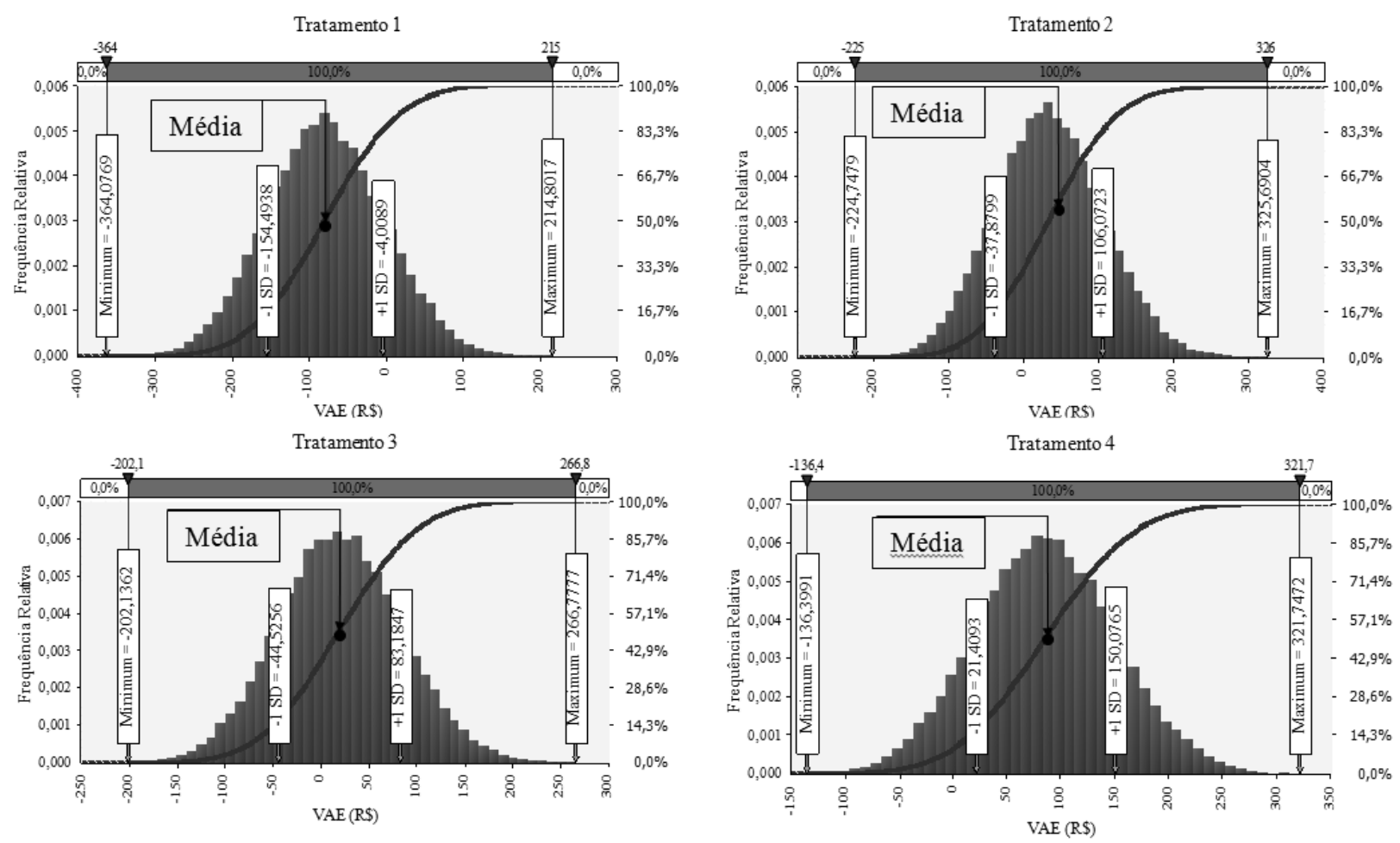

Figura 2 - Distribuição de frequência relativa e acumulada, VAEs máximos e mínimos e desvio padrão em relação à média, para os tratamentos

Figure 2 - Distribution of relative frequency and cumulative, EAV maximum and minimum and standard deviation from the average for treatments

de - R\$ 136,39 a R\$321,74. O VAE esperado ou médio está indicado pelo círculo, mostrando a probabilidade de ocorrerem valores maiores $(49,5 \%)$ e menores $(50,5 \%)$ do que a média na curva de frequência acumulada.

Os VAEs esperados dos tratamentos 2 e 3 são iguais a $\mathrm{R} \$ 34,10$ e $\mathrm{R} \$ 19,33$, respectivamente. Portanto, de acordo com o valor médio do VAE, ambos são viáveis economicamente, já que esses valores são positivos. Entretanto, a avaliação sob condições de incerteza é relativa, tendo em vista que houve uma dispersão considerável dos dados em torno da média, expressa pelo desvio padrão, tendo como consequência desfavorável a ocorrência de VAEs negativos, sendo seu valor mínimo de - R\$ 225,00 no tratamento 2 e de - R\$202,10 no tratamento 3 .

Como mostrado na Figura 2, uma diminuição equivalente a um desvio padrão no VAE dos tratamentos 2 e 3 é suficiente para que os mesmos se inviabilizem economicamente, já que, nessa condição, ambos teriam
VAEs negativos. Por outro lado, no tratamento 4 é preciso haver variação de mais de um desvio padrão no VAE, para que o mesmo seja negativo, mostrando que esse tratamento está menos sujeito a riscos que os demais.

\section{CONCLUSÕES}

O plantio de candeia em espaçamentos maiores é mais lucrativo e com menor risco de obter resultados desfavoráveis do que o plantio em espaçamentos menores. Assim, o espaçamento $1,5 \times 3,0 \mathrm{~m}$ é preferível aos espaçamentos $1,5 \times 1,5 \mathrm{~m}, 1,5 \times 2,0 \mathrm{~m}$ e $1,5 \times 2,5 \mathrm{~m}$.

Para os espaçamentos $1,5 \times 3,0 \mathrm{~m}, 1,5 \times 2,5 \mathrm{~m}, 1,5$ x 2,0 m e 1,5 x 1,5 m, a idade ótima de corte é de 12, 13, 13 , e 15 anos, respectivamente.

As informações obtidas acerca dos riscos econômicos envolvidos no plantio de candeia servem como ferramenta de auxílio na tomada de decisões em relação a novos plantios dessa espécie e também como

Cerne, Lavras, v. 20, n. 1, p. 113-122, jan./mar. 2014 
base para futuros experimentos com a mesma, visando o aprimoramento de seu cultivo.

\section{AGRADECIMENTOS}

A primeira autora agradece à Capes pela concessão da bolsa de estudos e o segundo autor agradece ao Conselho Nacional de Desenvolvimento Científico e Tecnológico $\mathrm{CNPq}$ - Brasil, pela bolsa de produtividade em pesquisa, e à Fapemig, pelo apoio financeiro por intermédio do Programa Pesquisador Mineiro - PPM IV (Processo CAG - PPM - 00286-10).

\section{REFERÊNCIAS}

ASSEMBLÉIA LEGISLATIVA DE MINAS GERAIS. Caracterização dos municípios mineiros. Disponível em: $<$ http://www.almg.gov.br/index BENTES-GAMA, M. M. et al. Análise econômica de sistemas agroflorestais na Amazônia Ocidental, Machadinho D’Oeste, RO. Revista Árvore, Viçosa, MG, v. 29, n. 3, p. 401-411, maio/jun. 2005.

CASTRO, C. de et al. Análise econômica do cultivo e extração do óleo essencial de Melaleuca alternifólia Cheel. Revista Árvore, Viçosa, MG, v. 29, n. 2, p. 241249, mar./abr. 2005.

CASTRO, R. R. de et al. Rentabilidade econômica e risco na produção de carvão vegetal. Cerne, Lavras, v. 13, n. 4, p. 353-359, out./dez. 2007.

COELHO JÚNIOR, L. M.; REZENDE, J. L. P. de; OLIVEIRA, A. D. de; COIMBRA, L.A.B.; SOUZA, A.N. de . Análise de investimento de um sistema agroflorestal sob situação de risco. Cerne, Lavras, v. 14, n. 4, p. 368-378, out./dez. 2008.

CORDEIRO, S. A.; SILVA, M. L. da. Rentabilidade e risco de investimento na produção de palmito de pupunha (Bactris gasipaes Kunth). Cerne, Lavras, v. 16, n. 1, p. 53-59, jan./mar. 2010.

HACURA, A.; JAMADUS-HACURA, M.; KOTOT, A. Risk analysis in investment appraisal based on the Monte Carlo simulation technique. European Physical Journal B, New York, v. 20, n. 4, p. 551-553, Apr. 2001. INSTITUTO BRASILEIRO DE GEOGRAFIA E

Cerne, Lavras, v. 20, n. 1, p. 113-122, jan./mar. 2014
ESTATÍSTICA. Carrancas, MG: dados básicos. Disponível em: $<$ http://www.ibge.gov.br/cidadesat/ painel/painel.php?codmun=311460 $>$. Acesso em: 18 jan. 2011.

KALLIO, A. M. I. Accounting for uncertainty in a forest sector model using Monte Carlo simulation. Forest Policy and Economics, Amsterdam, v. 12, n. 1, p. 9-16, Jan. 2010.

KNOKE, T.; MOOG, M.; PLUSCZYK, N. On the effect of volatile stumpage prices on the economic attractiveness of a silvicultural transformation strategy.

Forest Policy and Economics, Amsterdam, v. 2, n. 3/4, p. 229-240, July 2001.

LAPPONI, J. C. Projetos de investimentos na empresa. Rio de Janeiro: Elsevier, 2007. 332 p. MENDES, M. H.; SOUZA, R. C. Análise quantitativa de risco: um guia para modelagem pela simulação de Monte Carlo. Rio de Janeiro: PUC-RJ, 2007. 35 p.

OLIVEIRA, A. D. de et al. Economic analysis of sustainable management of candeia. Cerne, Lavras, v. 16, n. 3, p. 335-345, jul./set. 2010.

PALISADE CORPORATION. @Risk: risk analysis add-in for Excel. Version 5.5. New York, 2009. Software.

PÉREZ, J. F. M. Sistema de manejo para candeia (Eremanthus erythropappus (DC.) MacLeish). 2001. 71 p. Dissertação (Mestrado em Produção Florestal) Universidade Federal de Lavras, Lavras, 2001.

REZENDE, J. L. P. de; OLIVEIRA, A. D. de. Análise econômica e social de projetos florestais. 2. ed. Viçosa, MG: UFV, 2008. 386 p.

SECURATO, J. R. Decisões financeiras em condições de risco. São Paulo: Saint Paul, 2007. 264 p.

SILVA, C. P. de C. Crescimento e produção da candeia em plantio sujeito a diferentes espaçamentos e podas. 2009. 132 p. Dissertação (Mestrado em Engenharia Florestal) - Universidade Federal de Lavras, Lavras, 2009.

SOUZA, J. L. M. de. Modelo para a análise de risco 
econômico aplicado ao planejamento de projetos de irrigação para cultura do cafeeiro. 2001. 253 p.

Tese (Doutorado em Agronomia) - Escola Superior de Agricultura "Luiz de Queiroz", Piracicaba, 2001.

Recebido: 1 de junho de 2012; aceito: 15 de agosto de 2013. 
